\title{
The OGLE search for microlensing events towards the LMC
}

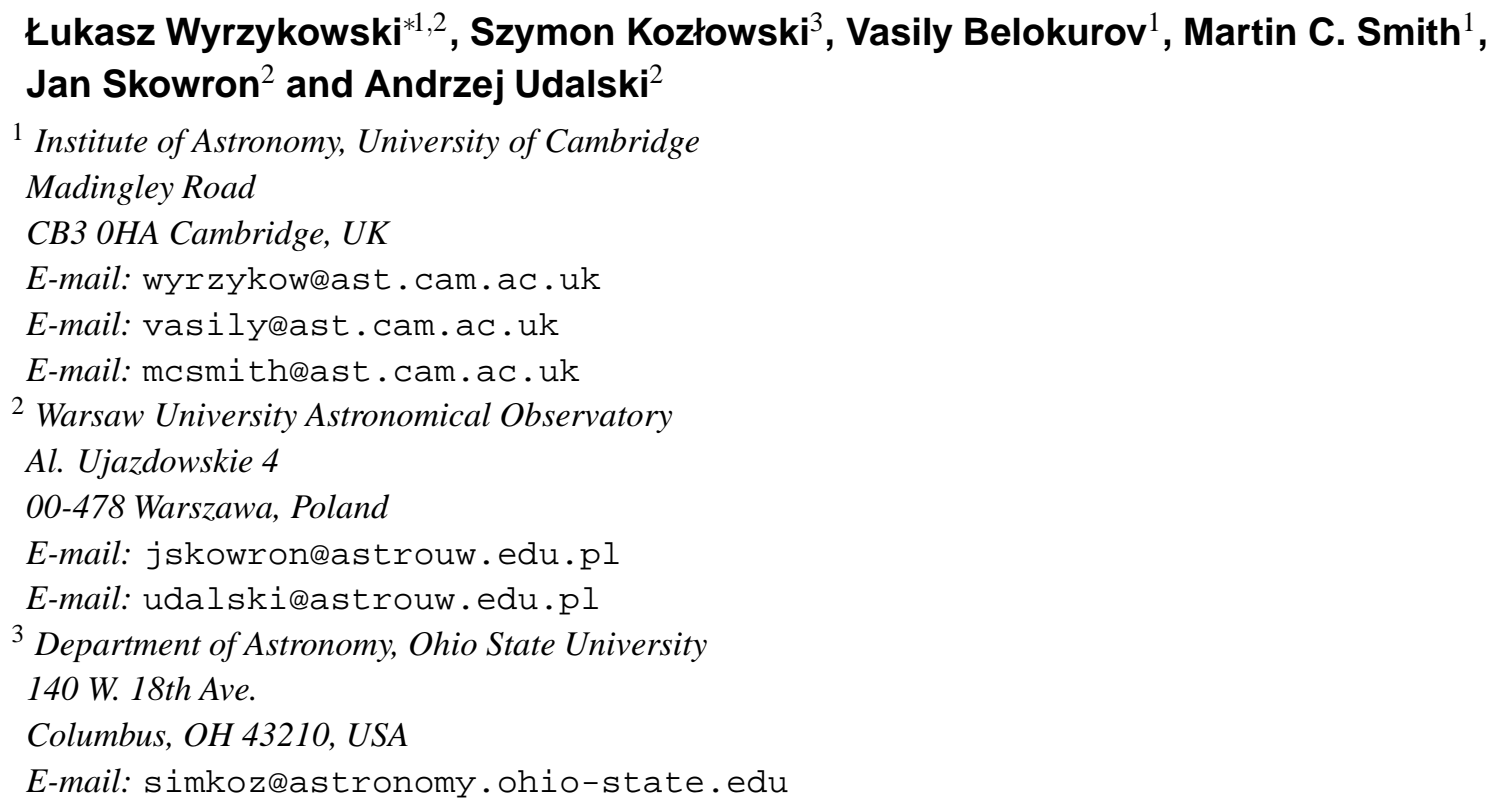

${ }^{1}$ Institute of Astronomy, University of Cambridge

Madingley Road

CB3 OHA Cambridge, UK

E-mail: wyrzykoweast.cam.ac.uk

E-mail: vasilyeast.cam.ac.uk

E-mail: mcsmitheast.cam.ac.uk

${ }^{2}$ Warsaw University Astronomical Observatory

Al. Ujazdowskie 4

00-478 Warszawa, Poland

E-mail: jskowroneastrouw.edu.pl

E-mail: udalskieastrouw.edu.pl

${ }^{3}$ Department of Astronomy, Ohio State University

140 W. 18th Ave.

Columbus, $\mathrm{OH} 43210$, USA

E-mail: simkoz@astronomy.ohio-state.edu

\begin{abstract}
We present results from the OGLE survey towards the Large Magellanic Cloud, which aimed to detect the microlensing phenomena caused by compact objects in the Galactic Halo (MACHOs). The analysed data covers nearly 10 years from 1997 to 2006 and includes the second and third phase of the OGLE project. In total about 35 million objects were monitored over an area of 38 sq.deg. The small number of plausible microlensing candidates indicates that the Galactic Halo towards the $\mathrm{LMC}$ is not made of dark matter in the form of MACHOs.
\end{abstract}

The Manchester Microlensing Conference: The 12th International Conference and ANGLES Microlensing Workshop

January 21-25 2008

Manchester, $U K$

\footnotetext{
${ }^{*}$ Speaker.
} 


\section{Introduction}

In 1986 Bohdan Paczyński [8] suggested using microlensing phenomenon for detection of massive compact halo objects, named MACHOs. He estimated that if such objects existed and had masses $M>10^{-6} M_{\odot}$ they should produce several microlensing events per year among millions of stars of the Large and Small Magellanic Clouds (LMC and SMC). His hypothesis triggered several microlensing surveys to start regular monitoring of stars in the Clouds in 1992. The results, however, have not yet proved conclusive.

MACHO group has analysed 5.7 years of their data and presented 17 candidate microlensing events [1]. A subsequent re-analysis of the same dataset constrained this sample to 10 events [3]. The resulting optical depth is $\tau=1.0 \pm 0.3 \cdot 10^{-7}$. The EROS collaboration has been observing a larger area of the sky than MACHO group for 6.7 years. They, however, found no microlensing candidates and they were only able to estimate the upper limit of the optical depth as $\tau<0.36 \cdot 10^{-7}$ [10]. This large discrepancy is thought to be due to different sample of stars used in these groups' analyses: MACHO used all stars, whereas EROS used only bright giants. Also MACHO's sample may be contaminated with variable stars, novae and supernovae [2].

In this paper we present the results and analysis of the independent dataset gathered by the OGLE project.

\section{Observational data}

Optical Gravitational Lensing Experiment (OGLE) has been monitoring the LMC since its second phase (OGLE-II) and continued to do so during the third phase (OGLE-III). Since 1996 OGLE has been using a dedicated $1.3 \mathrm{~m}$ telescope based in Las Campanas Observatory in Chile, operated by Carnegie Institution of Washington. During OGLE-II phase observations were taken with $2 \mathrm{k} \times 2 \mathrm{k}$ single CCD working in drift-scan mode allowing for single field of view of $0.21 \mathrm{sq}$. $\operatorname{deg}$ (14'x54'). Since 2001, in the third phase, the camera was replaced with mosaic of 8 CCD chips of $2 \mathrm{k} \times 4 \mathrm{k}$ each with total field of view of $0.34 \mathrm{sq}$. deg (35' $\left.\times 35^{\prime}\right)$. Details on instrumentation, set-up and photometry pipeline can be found in [11].

Total area covered by OGLE-II and OGLE-III was about 5 and 40 sq. deg, respectively. For comparison, MACHO collaboration analysed data from the area roughly as three times bigger than OGLE-II (about 14 sq. deg.) and EROS monitored and studied area nearly twice of that of OGLEIII (about 90 sq.deg).

\section{Search procedure and candidate events}

There were about 5.3 million objects in the OGLE-II database and 26.1 million in OGLE-III. Data from both phases were searched independently. Only the I-band photometry was investigated as it was far more numerous than V-band. The V-band data were only used for creating ColourMagnitude Diagrams (CMDs).

All light curves were first searched for a significant bump over any type of baseline, in a manner similar to [9]. Then a microlensing model was fit to each light curve and, based on the resulting parameters, the following series of cuts were applied: 
- both blended and non-blended models' fits converged

- magnification peak $\left(t_{0}\right)$ within the data range

- event's time-scale for the blended fit was within the range $1<t_{\mathrm{E}}<500 d$ for OGLE-II and $1<t_{\mathrm{E}}<750 d$ for OGLE-III

- impact parameter in blended model $0<u_{0} \leq 1$

- blending fraction $f_{\mathrm{S}}=\frac{F_{\text {source }}}{F_{\text {total }}}<1.2$, allowing for some negative blending

- number of data points at the peak (defined as $\left.t_{0} \pm 1 t_{\mathrm{E} \_ \text {nobl }}\right), N_{\text {peak }}>7$

- microlensing fit significantly better than constant line fit: $\frac{\chi_{c t}-\chi_{\mu}}{\chi_{\mu}} \frac{N}{\sqrt{2 N_{\text {peak }}}}>220$

- microlensing fit around the peak: $\frac{\chi_{\mu p e a k}}{N_{d o f}}<-2.5\left(I_{\max }-20.0 \mathrm{mag}\right)$

- microlensing fit better than supernova fit (described by an instantaneous rise to peak brightness followed by a decline modelled by two asymmetric exponentials) and number of datapoints before the peak at least $1 / 3$ of number of datapoints after the peak, $\frac{N_{\text {before_peak }}}{N_{\text {after_peak }}}>\frac{1}{3}$

The parameters of the above cuts were derived empirically based on Monte Carlo simulations of the events. The remaining cuts were related to the colour and magnitude of the source:

- magnitude limit: $I \leq 20.4 \mathrm{mag}$ (OGLE-II), $I \leq 21 \mathrm{mag}$ (OGLE-III), $I \leq 18.8 \mathrm{mag}$ (Bright Sample OGLE-II and OGLE-III)

- "blue bumper" cut: $V-I>0.5 \mathrm{mag}$ or $I>19 \mathrm{mag}$, in order to exclude contamination with bright blue stars, exhibiting brightening episodes similar to microlensing.

Using the above search procedure, 2 candidate microlensing events were found in the OGLEII database and 1 in OGLE-III. Their full-span and zoomed-in light curves are shown in Fig. 1. Their parameters are listed in Tab. 1. Since the OGLE-III fields include all the OGLE-II fields, we cross-correlated the OGLE-II events with the available OGLE-III data to ensure their baselines are constant. We also cross-correlated our events with the MACHO database, which is available online $^{1}$ and confirmed that our events did not exhibit any additional brightening. The total baselines for our three events were around 15 years, making these candidates strong.

Interestingly, none of our candidate events overlap with events detected by the MACHO collaboration ([1],[3]). In the case of OGLE51653.26-691630.1 and OGLE52449.21-675004.6 the reason is simply because these events occurred outside the data used in their analysis ${ }^{2}$. The MACHO data for the remaining event OGLE53048.00-695433.6 is very sparse at the time of the event and there is some small visible excursion from the baseline. This event very likely did not pass the MACHO selection criteria. Since MACHO photometry was obtained in multiple filters, one can use the MACHO data to test whether the event is chromatic. Although there is limited MACHO

\footnotetext{
${ }^{1}$ http://wwwmacho.anu.edu.au/Data/MachoData.html

${ }^{2}$ Actually, the whole magnification peak of event OGLE51653.26-691630.1 is present in the MACHO data, but this part of data was not studied by this collaboration.
} 

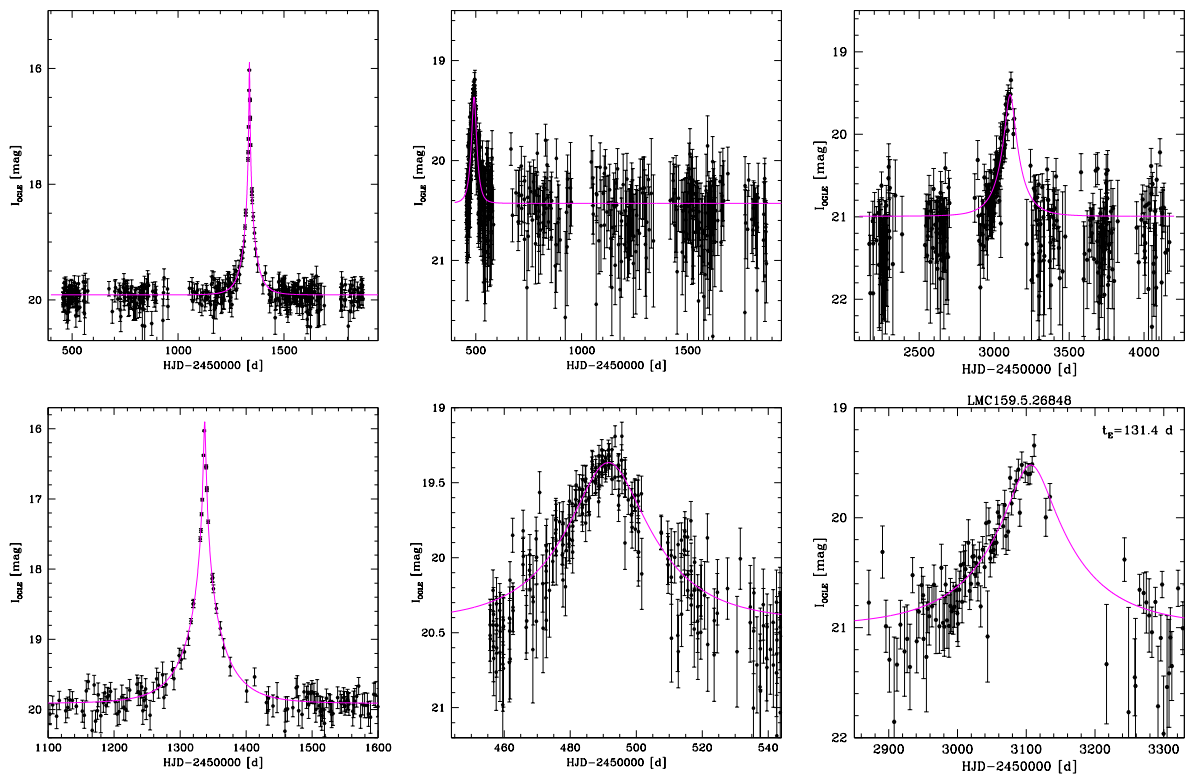

Figure 1: Lightcurves of OGLE-II events (left and middle) and OGLE-III event (right). Top panels show the full-span light curves, whereas bottom ones show zoom-ins around the peak. Magenta line is the best fitted point-lens microlensing model.

\begin{tabular}{|c|c|c|c|c|c|c|c|c|c|}
\hline lensed star name & $\begin{array}{c}\text { RA } \\
{[\mathrm{J} 2000.0]}\end{array}$ & $\begin{array}{c}\text { Dec } \\
{[\mathrm{J} 2000.0]}\end{array}$ & $\begin{array}{c}t_{0} \\
\text { [HJD-245000] }\end{array}$ & $\begin{array}{c}t_{\mathrm{E}} \\
\text { [days] }\end{array}$ & $u_{0}$ & $f_{\mathrm{S}}$ & $\begin{array}{c}I_{0} \\
{[\mathrm{mag}]}\end{array}$ & $\begin{array}{c}V_{0} \\
{[\mathrm{mag}]}\end{array}$ & $\begin{array}{l}\text { OGLE } \\
\text { phase }\end{array}$ \\
\hline OGLE51653.26-691630.1 & $5: 16: 53.26$ & $-69: 16: 30.1$ & $\begin{array}{c}1337.20 \\
\pm 0.02\end{array}$ & $\begin{array}{l}57.2 \\
\pm 4.6\end{array}$ & $\begin{array}{c}0.0258 \\
\pm 0.0025\end{array}$ & $\begin{array}{c}1.04 \\
\pm 0.09\end{array}$ & $\begin{array}{l}19.91 \\
\pm 0.01\end{array}$ & $\begin{array}{l}20.68 \\
\pm 0.14\end{array}$ & II \\
\hline OGLE53048.00-695433.6 & $5: 30: 48.00$ & $-69: 54: 33.6$ & $\begin{array}{l}491.6 \\
\pm 0.3\end{array}$ & $\begin{array}{l}24.2 \\
\pm 5.7\end{array}$ & $\begin{array}{c}0.4048 \\
\pm 0.1442\end{array}$ & $\begin{array}{c}1.02 \\
\pm 0.49\end{array}$ & $\begin{array}{l}20.40 \\
\pm 0.02\end{array}$ & $\begin{array}{l}20.64 \\
\pm 0.13\end{array}$ & II \\
\hline OGLE52449.21-675004.6 & $5: 24: 49.21$ & $-67: 50: 04.6$ & $\begin{array}{c}3106.5 \\
\pm 2.9\end{array}$ & $\begin{array}{r}132.4 \\
\pm 41.7\end{array}$ & $\begin{array}{c}0.2279 \\
\pm 0.1058\end{array}$ & $\begin{array}{c}0.83 \\
\pm 0.44\end{array}$ & $\begin{array}{l}20.99 \\
\pm 0.02\end{array}$ & $\begin{array}{l}22.32 \\
\pm 0.46\end{array}$ & III \\
\hline
\end{tabular}

Table 1: Microlensing event candidates detected in OGLE data. $I_{0}$ and $V_{0}$ stand for baseline magnitude in I and $\mathrm{V}$ band, respectively.

data available for the peak of this event, MACHO R- and B-bands may indicate some chromaticity. This requires further investigation.

Additionally we have checked if the MACHO candidate events are present in our data. Among 25 candidates of [1] only 9 were located within OGLE-II fields. For all of these nine events, the microlensing amplification does not occur during the 2 year overlap when both OGLE-II and MACHO were in operation.

Fig.2 shows the total (i.e. source plus blend) baseline magnitude and colour of all three events on the CMD and position on the sky, along with MACHO candidate events from [3]. Different symbols are used to differentiate between standard events, a binary event, self-lensing candidates (after [7]) and confirmed Galactic thick-disk events (\#5 and \#20 [6]). Note that both OGLE-II events are located centrally on the map showing Red Clump stellar density as the background. This favours an interpretation of them being caused by self-lensing due to stars in the LMC. On the other hand, the OGLE-III event clearly lies off from the populations of LMC stars on the CMD and is relatively red. Also, its location in the vicinity of the two MACHO events caused by thick-disk lenses suggests this could be another example of a Galactic lens towards the LMC. 

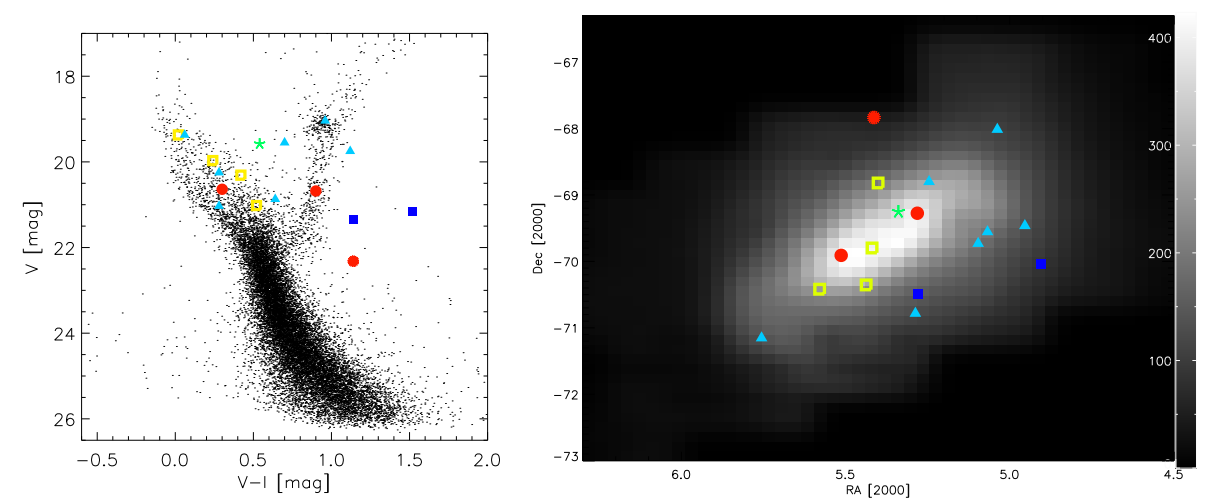

Figure 2: Left: CMD showing MACHO events and OGLE events, with HST data shown in the background (for a field centered on RA=81.0 deg, Dec=-68.8 deg (J2000); taken from [5]). Right: The spatial locations of OGLE and MACHO events overlaid onto the LMC Red Clump density map (in units of no.of RC per sq. arcmin). OGLE events are marked with red filled circles, with OGLE-III as a red star-like circle. Remaining symbols represent MACHO events: binary event \#9 (green star); candidates for self-lensing (yellow open squares); confirmed Galactic thick-disk lenses \#5 and \#20 (dark blue filled squares); and remaining candidates marked with blue triangles.

\section{Blending}

Dealing with blending requires special attention in optical depth calculations. Several authors have studied its influence. One approach is to try and avoid it, for example by analysing bright source stars in the belief that such samples should be free from blending (e.g. [10]). In our study we used high resolution Hubble Space Telescope (HST) images available in its on-line archive ${ }^{4}$. We selected deep I-band HST images overlapping with OGLE fields for various levels of stellar density. For each OGLE object in a field we identified corresponding HST stars with a limiting magnitude of $3 \mathrm{mag}$ below the OGLE object's magnitude. From this it is possible to construct distributions for the blending parameter and estimate the number of stars within the seeing disc of each OGLE object.

We divided OGLE fields into 5 density levels, from very sparse to very dense (dividing levels at 100,200,350 and 600 stars per sq.arcmin). For each density level we obtained the aforementioned distributions, dividing objects into three magnitude bins: 14-17.5, 17.5-19 and below 19 mag. The distribution of the number of HST stars corresponding to each OGLE object was then convolved with the luminosity function of each field, according to its density level. This resulted in an estimate of the real number of monitored stars. For OGLE-II data this yielded 9.6 million stars (for 5.3 million objects in the database), while for OGLE-III this gave 47.7 million stars (for 26.1 million objects in the database). We performed this calculation also for the Bright Sample (see section 6.2) and found that for OGLE-II the real number of stars was 2.8 million (1.8 in the database) and for OGLE-III it was 7.8 million (5.5 in the database).

\footnotetext{
${ }^{4}$ http://archive.stsci.edu/hst/
} 


\begin{tabular}{ccccc} 
event & $t_{\mathrm{E}}$ & $\varepsilon\left(t_{\mathrm{E}}\right)^{\dagger}$ & $\tau_{i} \cdot 10^{-7}$ & \\
\hline OGLE53048.00-695433.6 & $24.2 \pm 5.7$ & 0.1404 & 0.20 & \\
OGLE51653.26-691630.1 & $57.2 \pm 4.6$ & 0.2301 & 0.28 & \\
total $\tau_{O G L E-I I}$ & & & & $0.48 \pm 0.37$ \\
\hline $\begin{array}{c}\text { OGLE52449.21-675004.6 } \\
\text { total } \tau_{O G L E-I I I}\end{array}$ & $132.4 \pm 41.7$ & 0.1710 & 0.12 & \\
\hline † detection efficiency corrected for non-detectability of binary events
\end{tabular}

Table 2: Optical depth values for the All Stars sample.

\section{Detection efficiency}

We performed Monte Carlo simulations in order to obtain the detection efficiency for microlensing events. Although we have done this on the catalogue level, we have tried to reproduce all factors that are present in image-level simulations.

We randomly picked a star with all its photometric measurements. For fixed $t_{\mathrm{E}}$, the rest of microlensing parameters were drawn as follows: $t_{0}$ and $u_{0}$ from flat distributions; $f_{\mathrm{S}}$ from the distribution of blending parameter (ratio of source star flux to the total flux). As noted above, the $f_{\mathrm{S}}$ distribution depends on the stellar density and baseline magnitude, which can be determined for the randomly selected star. Then, the original flux of the OGLE object was divided between the source and the blend, according to the blending parameter $f_{\mathrm{S}}$. Note that this approach does not involve the addition of any 'fake' stars, i.e. we microlensing existing stars in the image. Our procedure also preserved any variability and non-gaussian scatter present in the original photometry.

\section{Optical Depth}

We determined the optical depth for two different approaches: using all stars (following the MACHO collaboration) and using only bright stars (following the EROS collaboration).

\subsection{All Stars}

In our All Stars sample there were 2 candidate events found in OGLE-II data and 1 candidate event in OGLE-III. We used the following standard equation for calculating the optical depth:

$$
\tau=\frac{\pi}{2 N_{*} T_{o b s}} \sum_{i}^{N_{e v}} \frac{t_{\mathrm{E} i}}{\varepsilon\left(t_{\mathrm{E} i}\right)}
$$

where $T_{o b s}$ is total exposure time, $N_{*}$ is total number of monitored stars, $N_{e v}$ is total number of events, $t_{\mathrm{E} i}$ is the time-scale of each event detected with an efficiency of $\varepsilon\left(t_{\mathrm{E} i}\right)$. We calculated the optical depth separately for events detected in each OGLE phase, using respective exposure times of $T_{I I}=1428$ days and $T_{I I I}=2058$ days) and blending-corrected number of monitored stars $N_{I I}=$ $9.6 \cdot 10^{6}$ and $N_{I I I}=47.7 \cdot 10^{6}$ (see section 4). The time-scales of the events used here were obtained by fitting the microlensing curve with blending as a free parameter. Efficiencies obtained through the Monte Carlo simulations was additionally multiplied by 0.9 to account for non-detectability of binary events. Table 2 presents all the calculations of the optical depth. The error in $\tau$ was calculated using the formula given by [4]. 


\subsection{Bright Sample}

We also followed the approach presented by EROS collaboration [10], selecting stars brighter than $I=18.8$ mag (cut just below the Red Clump Giants), taking into account slight shifts due to extinction. We applied the criteria described in section 3 to the Bright Sample and from this we have not found any microlensing candidates in either OGLE-II or OGLE-III data. Since we have no candidates, we can only place an upper limit to the optical depth. Followed the analysis of [10], we estimated the number of expected microlensing events for a standard Galaxy halo model consisting of $100 \%$ MACHOs. This, combined with the detection efficiency for different time-scales, resulted in an upper limit to the fraction of MACHOs in the halo.

We performed our analysis for two cases: firstly, assuming no blending, as in [10]; and, secondly, taking blending into account in the same manner as for the All Star sample. In the non-blended approach, for a typical MACHO mass of $0.4 M_{\odot}$, the number of expected events in OGLE-II and OGLE-III is around 6 and 28, respectively. Hence we expect a combined total of 34 events in both datasets from the total of 7.3 million stars and the total time of observations of 9.5 years. When blending is taken into account we expect 7 and 30 events in OGLE-II and OGLE-III, respectively, among the total number of stars of 11.2 million.

These numbers led to an upper limit on the optical depth due to sub-solar mass MACHOs of

$$
\begin{gathered}
\tau_{\text {non-blended }}<0.45 \pm 0.0510^{-7}\left[1+\log \left(M / 0.4 M_{\odot}\right)\right], \quad f_{\text {non-blended }}<0.09\left[1+\log \left(M / 0.4 M_{\odot}\right)\right] \\
\tau_{\text {blended }}<0.41 \pm 0.0510^{-7}\left[1+\log \left(M / 0.4 M_{\odot}\right)\right], \quad f_{\text {blended }}<0.08\left[1+\log \left(M / 0.4 M_{\odot}\right)\right]
\end{gathered}
$$

where $f$ is a fraction of MACHOs in the halo.

Fig. 3 shows number of expected events in OGLE-II, OGLE-III and the combined data as a function of mass of the MACHO and the upper limit on the MACHO halo fraction for the combined OGLE-II and OGLE-III data, along with results from the MACHO and EROS collaborations. Results obtained with blended and non-blended approach are nearly the same with the only difference at small mass end due to lower detection efficiency of events with very short time-scales $\left(t_{\mathrm{E}}<7\right.$ days $)$.
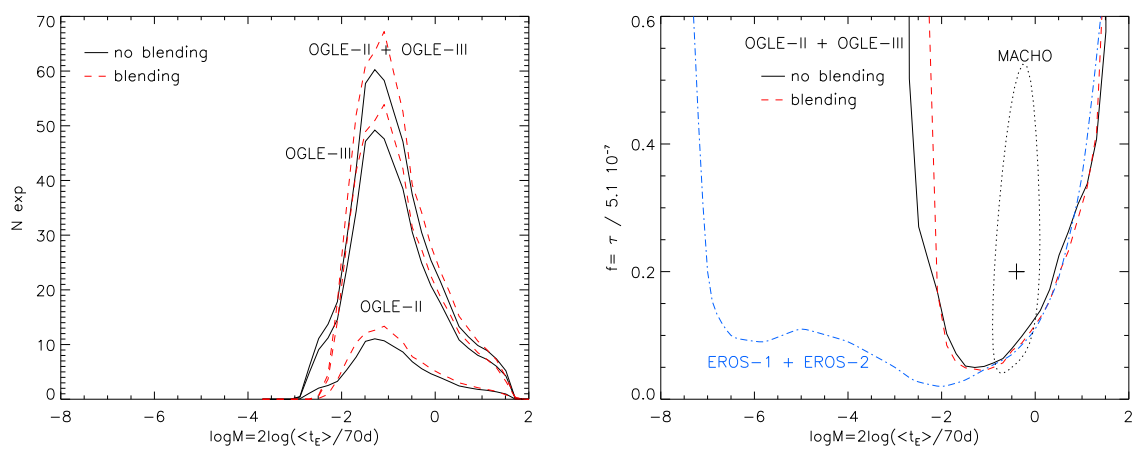

Figure 3: Number of expected events in Bright Sample (left) and the upper limit on the MACHO halo fraction (right). Results from the MACHO (black dotted) and EROS (blue dash-dotted) are also shown for comparison. Different curves show OGLE results for blended and non-blended approach. 
For MACHO masses higher than $0.1 M_{\odot}$ the OGLE result overlaps very well with the upper limit derived by the EROS collaboration. There is no limit from OGLE data for masses lower than $0.1 M_{\odot}$ due to the fact that OGLE sampling is sparser than EROS.

\section{Conclusions}

In the OGLE data spanning years 1997 to 2006 we found a total of 3 candidate microlensing events in the All Stars sample and none in the Bright Sample. Values of the optical depth were calculated for the events found in the OGLE-II and OGLE-III data separately. These are in agreement with the upper limit obtained with Bright Stars sample.

These results are also in agreement with results published by EROS collaboration ([10]) and within $1-\sigma$ of the value estimated by MACHO group ([3]). Our Bright Sample upper limit, as a function of a MACHO mass, is also in excellent agreement with the limit given by the EROS group for masses higher than $0.1 M_{\odot}$. We have also shown that for samples of bright stars, neglecting blending is fully justified in the analysis of the Large Magellanic Cloud.

We believe that our results are consistent with a scenario of self-lensing either within the LMC or our Galaxy, although the events we have found require further detailed investigation in order to confirm the self-lensing hypothesis. However, this provides strong indication that the vast majority of the Galactic halo cannot be in the form of massive compact objects. Even is one of our candidates turns out to be false, then our claim about MACHO-less halo will be reinforced.

\section{Acknowledgments}

This work was supported by the European Community's FR6 Marie Curie Programme, Contract No. MRTN-CT-2004-505183 “ANGLES". OGLE project is partially supported by the Polish MNiSW grant N20303032/4275.

\section{References}

[1] Alcock C., Allsman R. A., Alves D. R., et al., 2000, ApJ, 542, 281

[2] Belokurov V., Evans N.W., Du Y.L. 2003, MNRAS, 341, 1373

[3] Bennett D. P., 2005, ApJ, 633, 906

[4] Han C., Gould A., 1995, ApJ, 449, 521

[5] Holtzman, J., Afonso, C., \& Dolphin, A., 2006, ApJS, 166, 534

[6] Kallivayalil N. et al, 2006, ApJ, 652, L97

[7] Mancini L., Calchi Novati S., Jetzer P., Scarpetta G., 2004, A\&A, 427, 61

[8] Paczynski B., 1986, ApJ, 304, 1

[9] Sumi T., Woźniak P. R., Udalski A. et al., 2006, Astrophysical Journal, 636, 260

[10] Tisserand P. et al., 2006, AA submitted, astro-ph/0607207

[11] Udalski A., Kubiak M., Szymanski M., 1997, AcA, 47, 319

[12] Wyrzykowski L., Udalski A., Mao S., Kubiak M., Szymanski M. K., Pietrzynski G., Soszynski I., Szewczyk O., 2006, AcA, 56, 145 